\title{
Is the Lone Scientist an American Dream? Perceived Communal Opportunities in STEM Offer a Pathway to Closing U.S.-Asia Gaps in Interest and Positivity
}

Social Psychological and Personality Science 2018, Vol. 9(I) II-23

(C) The Author(s) 2017

Reprints and permission: sagepub.com/journalsPermissions.nav DOI: 10.1 I77/1948550617703।73 journals.sagepub.com/home/spp

(QSAGE

\author{
Elizabeth R. Brown', Mia Steinberg ${ }^{2}$, Yun Lu$^{3}$, \\ and Amanda B. Diekman ${ }^{4}$
}

\begin{abstract}
The United States lags behind many Asian countries in engagement in science, technology, engineering, and mathematics (STEM). An unexplored factor in these country-level differences may be U.S.-Asia gaps in perceptions of the goal opportunities provided by STEM. Across four studies, U.S. students perceived fewer communal opportunities (working with/helping/relationships with others) in STEM than Asian students; this differential perception contributed to U.S.-Asia gaps in STEM interest. Perceptions of communal opportunities in STEM did not follow from a general orientation to perceive that all careers provided communal opportunities but from communal engagement in STEM. Perceptions about communal opportunities in STEM predicted STEM interest, and communal experience in STEM predicted STEM interest beyond quantity of STEM exposure. Experimentally highlighting the perceived communal opportunities in science closed the cultural gap in positivity toward a scientist career (Study 5). Perceptions of communal opportunities in STEM provide a new vantage point to improve U.S. engagement in STEM.
\end{abstract}

\section{Keywords}

STEM, communion, motivation, culture, science education

Educational and economic advisories decry that the United States lags behind Asian countries' engagement in science and engineering (SE; National Science Board [NSB], 2014, 2016; President's Council of Advisors on Science and Technology [PCast], 2010; U.S. Congress Joint Economic Committee, 2012). In the first decade of the 21 st century, the number of bachelor's degrees awarded in SE doubled in China and quintupled in India, but only rose by $50 \%$ in the United States (NSB, 2012, 2016). Although social psychology most often speaks to the important goal of increasing the number of underrepresented group members in SE, social psychology can also speak to the important goal of increasing participation among both represented and underrepresented groups. Simply put, the United States needs greater numbers of students, across all demographic groups, engaged in science, technology, engineering, and mathematics (STEM). Although educators are exhorted to "prepare and inspire" students in STEM (PCast, 2010), it is difficult to find a foothold on this path (Hines, Mervis, Mccartney, \& Wible, 2013). We explore such a foothold by examining U.S.-Asia differences in beliefs about the goal opportunities provided by STEM careers.

Understanding why certain groups do or do not engage in STEM has led to a focus on beliefs about the nature and purpose of SE work. When students perceive science or math as useful, they are more likely to engage deeply and perform well (Eccles \& Wigfield, 1995; Harackiewicz, Smith, \& Priniski, 2016). Understanding how to harness a wide range of student motivations can provide explanatory power above and beyond self-efficacy or anticipated success (e.g., Valla \& Ceci, 2014). We apply goal congruity logic — that is, that people seek social roles that are perceived as providing valued goal opportunities (Diekman, Steinberg, Brown, Belanger, \& Clark, 2016) to understand cultural differences in the perceived goal opportunities in STEM roles, and in turn, U.S.-Asia gaps in STEM interest.

\footnotetext{
' Department of Psychology, University of North Florida, Jacksonville, FL, USA ${ }^{2}$ Academic Affairs, California State University, Long Beach, Long Beach, CA, USA

${ }^{3}$ Department of Counseling, Higher Education, and Special Education, University of Maryland, College Park, College Park, MD, USA

${ }^{4}$ Department of Psychology, Miami University, Oxford, OH, USA
}

Corresponding Author:

Elizabeth R. Brown, Department of Psychology, University of North Florida, Jacksonville, FL, USA.

Email: elizabeth.r.brown@unf.edu 
U.S. stereotypes about STEM include robust beliefs that STEM fields do not allow for the fulfillment of communal goals (Diekman, Brown, Johnston, \& Clark, 2010; Diekman, Clark, Johnston, Brown, \& Steinberg, 2011). Communion, which reflects orientation to others (e.g., working with, helping, and forming relationships with others), and agency, which reflects orientation to the self (e.g., achievement, self-promotion), are posited as two fundamental modalities of human psychology (Bakan, 1966; Pohlmann, 2001). The perceived lack of opportunity to fulfill communal goals in STEM can thus deter students. This communal goal congruity framework originated to understand the gender gaps in STEM career interest because of the gender differences that emerge in communal orientation (Diekman et al., 2010). However, the goal congruity logic applies beyond gender (Diekman et al., 2016) because communal goals reflect fundamental human needs to connect with others (Baumeister \& Leary, 1995; Fiske, 2004; Ryan \& Deci, 2000). Within the current research, we invoke a broad definition of communal goals in order to clearly contrast them with agentic goals, which have received more attention in explaining STEM engagement.

Given the high value accorded to communal goals, the extent that roles are perceived as incorporating communal opportunities should evoke positivity toward those roles. For instance, in the United States, perceptions that STEM does not provide communal opportunities are robustly associated with decreased interest, motivation, and positivity toward STEM careers (Brown, Thoman, Smith, \& Diekman, 2015; Thoman, Brown, Mason, Harmsen, \& Smith, 2015), whereas perceived agentic opportunities are only weakly associated with increased interest in STEM (Brown, Smith, Thoman, Allen, \& Muragishi, 2015; Smith, Brown, Thoman, \& Deemer, 2015). Importantly, the relationship between perceptions that STEM provides communal opportunities and STEM interest/ positivity holds when accounting for perceptions of the agentic opportunities provided by STEM (Brown, Smith, et al., 2015).

Emphasizing communal opportunities offers a new route to broadening the STEM pipeline by eliciting greater STEM interest. For instance, descriptions of a scientist's day involving collaborative work, relative to independent work, elevated interest in pursuing science (Clark, Fuesting, \& Diekman, 2016), especially among those who valued communal goals (Diekman et al., 2011). The benefit of highlighting the other-oriented nature of STEM activities has emerged in middle school engineering activities (Colvin, Lyden, \& León de la Barra, 2013), high school science demonstrations (Weisgram \& Bigler, 2006), college research experiences (Brown, Smith, et al., 2015), and computer science and engineering curricula (Brinkman \& Diekman, 2016). Exposure to communal opportunities in SE can disrupt stereotypic perceptions that these fields do not provide communal opportunities and foster positivity toward SE. The current research examines whether there is a U.S.-Asia gap in perceptions of the communal opportunities provided by STEM as well as whether counterstereotypic information can be leveraged within the United States to narrow this gap.
The potential for Asian and U.S. participants to differentially perceive communal opportunities in STEM may be rooted in cultural differences in social orientation, with greater interdependence among Asian individuals and greater independence among U.S. individuals (Markus \& Kitayama, 1991). The tendency to construe the self and objects as embedded within the whole has been traced to differential social structures within Asian societies that highlight collective interdependence (Talhelm et al., 2014; Uskul, Kitayama, \& Nisbett, 2008; Varnum, Grossmann, Kitayama, \& Nisbett, 2010). We examine three potential routes by which this collective interdependence might foster different perceived goal opportunities in STEM: (1) Asian, as compared with U.S., students might perceive more communal opportunities across all occupational roles, not limited to STEM; (2) Asian, as compared with U.S., students might take more STEM classes, fostering perceptions that STEM provides more communal and agentic opportunities; and (3) Asian, as compared with U.S., students might report experiencing more communal ways of engaging in STEM, fostering perceptions that STEM provides more communal opportunities.

Drawing on five cross-national samples using correlational and experimental methods, we demonstrate that U.S. and Asian participants (1) differ in their interest/positivity toward STEM and (2) differentially perceive that STEM provides communal, but not agentic, opportunities. We trace the sources of these communal opportunity perceptions and demonstrate the consequences of these sources for STEM interest and positivity. Studies 1-4 employed survey methods to examine the relationships among perceptions about the communal and agentic opportunities provided by STEM, experiences in STEM, and interest in STEM; Study 5 showed that experimentally elevated communal, but not agentic, opportunities in science can close the cultural gap in positivity toward a scientist career.

\section{Studies I-4}

Because the survey procedures of Studies 1-4 were similar, we present the method and results of these studies together and organize results by the key questions investigated.

\section{Method}

\section{Sample Size}

In each study, we collected at least 73 students from each country to detect a moderate effect size at .85 power. We oversampled to allow for weaker effect sizes and supplementary analyses (see Supplemental Material).

\section{Participants}

In Study 1, 192 native Indian citizens who were college students ( 82 women; ages $18-38$ [ $m d n=25]$ ) and 152 native U.S. citizens who were college students ( 89 women; ages $18-46[m d n=23])$ were recruited for payment using Mechanical Turk (MTurk). An additional three college students 
indicated dual Indian/U.S. citizenship (1 woman; ages 23-29 $[m d n=27])$ and were omitted from analyses. The majority of each sample (77.49\% Indian; $82.78 \%$ United States) were enrolled at a 4-year university.

In Study 2, 144 Chinese college students (84 women; ages $18-29[m d n=20])$ and 190 U.S. college students (148 women; ages $18-58[\mathrm{mdn}=21])$ participated for course credit. ${ }^{1}$ Both universities were selective public institutions with graduate schools.

In Study 3, 117 native Indian citizens who were college students (44 women; ages 19-35 [ $m d n=24]$ ) and 140 native U.S. citizens who were college students ( 84 women; ages $18-57$ $[m d n=24])$ were recruited for payment using MTurk. The majority of each sample $(72.65 \%$ Indian; $80.00 \%$ United States) were enrolled at a 4-year university.

In Study 4, 210 Chinese college students (118 women; ages $18-30[m d n=22])$ and 347 U.S. college students $(235$ women; ages $18-30[m d n=18]$ ) participated for course credit. ${ }^{1}$ Both universities were selective public institutions with graduate schools.

\section{Procedure}

Students completed an online survey in English that asked their perceptions about whether STEM careers provided communal and agentic opportunities and their interests in STEM careers. In Studies 1 and 3, students rated their perceptions about whether male-stereotypic and female-stereotypic careers provided communal and agentic opportunities. In Studies 3 and 4, students reported their communal engagement in STEM and how many STEM classes they had taken or were currently taking.

\section{Dependent Variables}

Communal engagement in STEM. In Studies 3 and 4, students rated their agreement with statements about their STEM experiences (e.g., "I worked on group projects in my science, technology, and mathematics courses"; Steinberg, 2011) on scales ranging from 1 (never) to 7 (very often). Items were averaged (Study 3, 10-item version: $\alpha=.94$; Study 4, 4-item version: $\alpha=.80$ ).

Number of STEM classes. In Studies 3 and 4, students reported how many math and SE classes they had taken or were currently taking.

Perceived communal and agentic opportunities. Students rated their perceptions that different STEM careers provided communal opportunities ("such as intimacy, affiliation, and altruism") and agentic opportunities ("such as power, achievement, and seeking new experiences or excitement;" Diekman et al., 2010) on scales ranging from 1 (not at all) to 7 (extremely). In Studies 1 and 3, students rated four STEM careers (mechanical engineer, computer scientist, aerospace engineer, and environmental scientist). In Studies 2 and 4, students rated two STEM fields: science and engineering. Items for perceived communal and agentic opportunities were separately averaged (Communion: Study $1: \alpha=.84$, Study 2 : $\alpha=.69$, Study $3: \alpha=.93$, Study $4: \alpha=.68$; Agency: Study 1 : $\alpha=.75$, Study $2: \alpha=.70$, Study $3: \alpha=.90$, Study $4: \alpha=.65$ ).

In Studies 1 and 3, students also rated their perceptions of whether male-stereotypic (dentist, lawyer, architect, and physician) and female-stereotypic (preschool teacher, registered nurse, ${ }^{2}$ human resources manager, social worker, and education administrator) careers provided communal and agentic opportunities (Diekman et al., 2010) on scales ranging from 1 (not at all) to 7 (extremely). Items within each scale were averaged (Communion: Study 1: $\alpha_{\mathrm{FSTER}}=.80$, $\alpha_{\text {MSTER }}=.65$, Study $3: \alpha_{\text {FSTER }}=.88, \alpha_{\text {MSTER }}=.89$; Agency: Study 1: $\alpha_{\mathrm{FSTER}}=.85, \alpha_{\mathrm{MSTER}}=.77$, Study 3 : $\alpha_{\mathrm{FSTER}}=.90, \alpha_{\mathrm{MSTER}}=.89$ ).

STEM career interest. Students rated their interest in different STEM careers on scales ranging from 1 (not at all) to 7 (extremely). In Studies 1 and 3, students rated the careers in the perceived opportunities measure as well as industrial engineer, chemical engineer, electrical engineer, and network and computer systems administrator (Diekman et al., 2010). In Studies 2 and 4, students rated their interest in SE careers on 14 items (e.g., How enjoyable would a science career be to you?; modified from Diekman et al., 2011). Items for STEM career interest were averaged (Study 1: $\alpha=.91$; Study 2: $\alpha=.91$; Study 3: $\alpha=.93$; Study $4: \alpha=.91$ ).

\section{Results}

After documenting the U.S.-Asia gap in STEM career interest, we examined whether U.S. and Asian students differentially perceived communal and agentic opportunities in STEM. Next, we examined whether any differential perceptions about goal opportunities predicted U.S.-Asia gaps in STEM interest; then, we examined the sources of perceived goal opportunities in STEM. For clarity, we report results relevant to the key questions here, and we provide complete analyses of variance (ANOVAs) in Tables 1-3. Analyses including gender are presented in the Supplemental Materials but do not affect the patterns and conclusions reported here.

\section{U.S.-Asia Gaps in STEM Career Interest}

For each study, we conducted a one-way ANOVA with country as a between-subjects variable. Asian students reported more interest in STEM careers than did U.S. students, Study 1: $F(1,342)=81.15, p<.001, d=0.98$, CI [.828, 1.134]; Study 2: $F(1,324)=13.92, p<.001, d=0.42$, CI [0.293, 0.542]; Study 3: $F(1,252)=53.83, p<.001, d=0.93$, CI [0.741, 1.116]; Study 4: $F(1,556)=83.39, p<.001, d=0.80, \mathrm{CI}$ [0.705, 0.892]. 
Table I. STEM Career Interest Ratings-Studies 1-4. ${ }^{\text {a }}$

One-Way ANOVAs With Country as a Between-Subjects Variable

\begin{tabular}{|c|c|c|c|c|c|c|c|c|c|c|}
\hline & \multicolumn{5}{|c|}{ Study I (df: I, 342) } & \multicolumn{5}{|c|}{ Study 2 (df: I, 324) } \\
\hline United States & - & - & - & 3.20 & 1.62 & - & - & - & 3.79 & 1.16 \\
\hline \multirow[t]{3}{*}{ China/India } & - & - & - & 4.62 & 1.31 & - & - & - & 4.27 & 1.15 \\
\hline & \multicolumn{5}{|c|}{ Study 3 (df: I, 252) } & \multicolumn{5}{|c|}{ Study 4 (df: I, 556) } \\
\hline & $F$ & $p$ & $d$ & $M$ & $S D$ & $F$ & $p$ & $d$ & $M$ & $S D$ \\
\hline Country & 53.83 & $<.001$ & .93 & - & - & 83.39 & $<.001$ & .80 & - & - \\
\hline United States & - & - & - & 2.91 & 1.61 & - & - & - & 3.54 & 1.20 \\
\hline China/India & - & - & - & 4.32 & $\mathrm{I} .44$ & - & - & - & 4.44 & 0.99 \\
\hline
\end{tabular}

Note. STEM = science, technology, engineering, and mathematics; ANOVAs = analyses of variance.

${ }^{a}$ Positive effect sizes (Cohen's $d$ ) indicate higher ratings for Asian students.

Table 2. Perceptions of Communal and Agentic Opportunities in STEM-Studies I-4. ${ }^{\mathrm{a}}$

2 (country: United States, China/India) $\times 2$ (perceived opportunities: communal, agentic) ANOVA

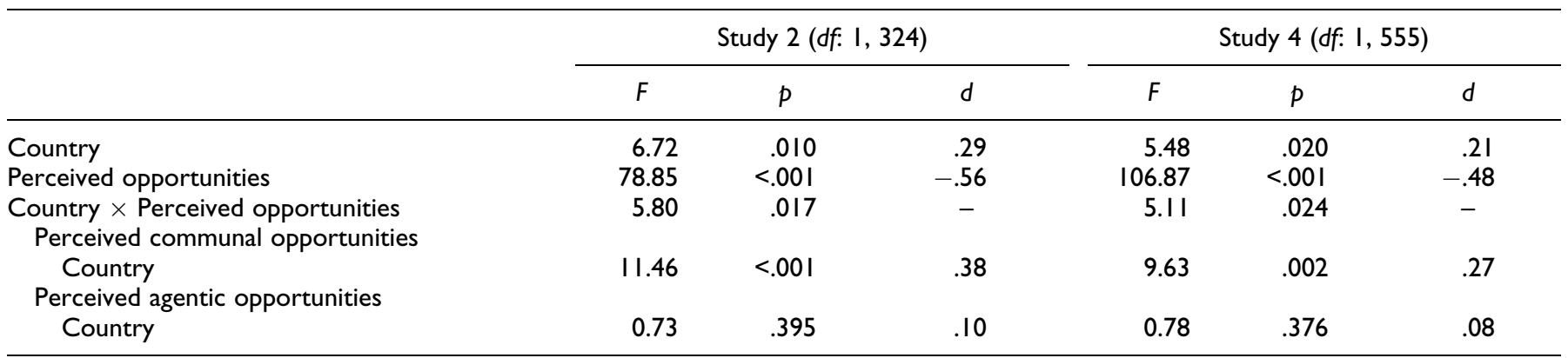

2 (country: United States, China/India) $\times 2$ (perceived Opportunities) $\times 3$ (career type: STEM, male-stereotypic, female-stereotypic) ANOVA

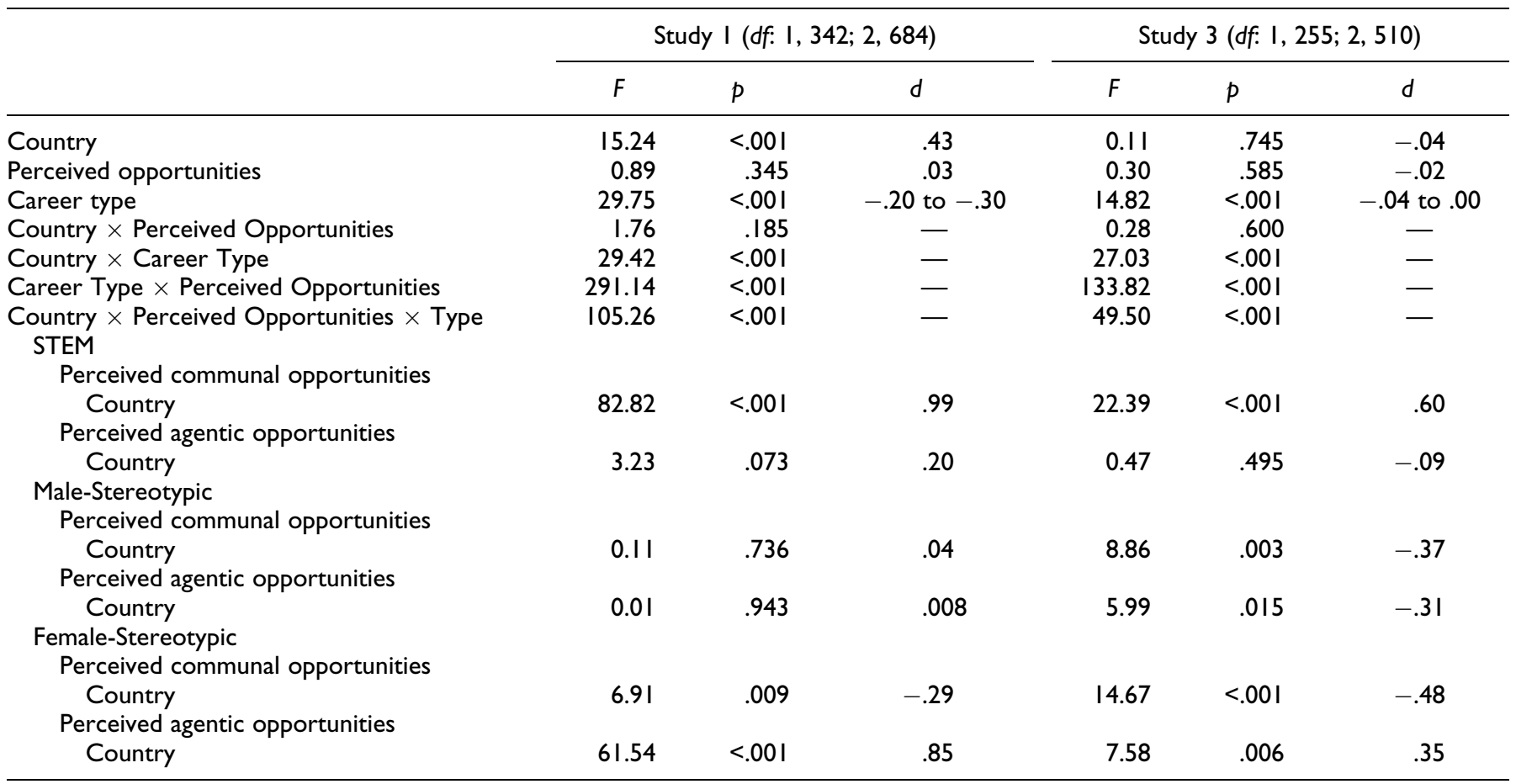

Note. STEM = science, technology, engineering, and mathematics; ANOVA = analysis of variance.

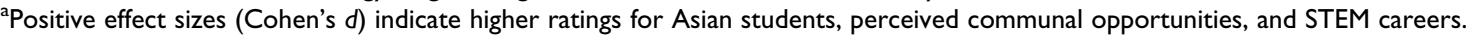


Table 3. Communal Engagement and Quantity of Experiences in STEM-Studies 3 and 4. ${ }^{\mathrm{a}}$

One-Way ANOVA With Country as a Between-Subjects Variable

\begin{tabular}{|c|c|c|c|c|c|c|c|c|c|c|}
\hline Communal Engagement & \multicolumn{5}{|c|}{ Study 3 (df: I, 25I) } & \multicolumn{5}{|c|}{ Study 4 (df: I, 555) } \\
\hline United States & - & - & 2014 & 3.02 & 1.67 & - & - & - & 2.67 & 1.43 \\
\hline China/India & - & - & - & 4.59 & 1.42 & - & - & - & 4.05 & 1.17 \\
\hline Country & 34.72 & $<.001$ & 0.76 & - & - & 32.26 & $<.001$ & 0.50 & - & - \\
\hline United States & - & - & - & II.8I & 10.01 & - & - & - & 8.46 & 6.43 \\
\hline China/India & - & - & - & 22.15 & 17.25 & - & - & - & 12.33 & 9.60 \\
\hline
\end{tabular}

Note. STEM = science, technology, engineering, and mathematics; ANOVA = analysis of variance.

${ }^{\text {a }}$ Positive effect sizes (Cohen's $d$ ) indicate higher ratings for Asian students.

\section{Did U.S. and Asian Students Differentially Perceive That STEM Careers Provided Communal and Agentic Opportunities?}

To explore whether Asian students perceived that STEM careers provided different communal and agentic opportunities than U.S. students, we conducted a 2 (country) $\times 2$ (perceived opportunities: communal, agentic) mixed ANOVA with perceived opportunities as a within-subjects variable. Across all studies, the predicted Country $\times$ Perceived Opportunities interaction emerged, Study 1: $F(1,342)=58.02, p<.001$; Study 2 : $F(1,324)=5.80, p=.017$; Study 3: $F(1,255)=32.07$, $p<.001$; Study 4: $F(1,555)=5.11, p=.024$. As shown in Figure 1, Asian students more than U.S. students perceived that STEM careers provided communal opportunities (Study 1: $p<.001, d=0.99$, CI [0.857, 1.125]; Study 2: $p<.001$, $d=0.38$, CI [0.241, 0.516]; Study 3: $p<.001, d=0.60$, CI $[0.424,0.766]$; Study 4: $p=.002, d=0.27$, CI $[0.171,0.373])$. In contrast, only one unpredicted and marginal effect emerged for perceived agentic opportunities: In Study 1, Asian students were marginally more likely than U.S. students to perceive that STEM careers provided agentic opportunities ( $p=.073, d=0.20$, CI $[0.071,0.320]$ ).

\section{Did Differential Perceptions About Communal and Agentic Opportunities Underlay the U.S.-Asia Gap in STEM Interest?}

Next, we examined whether the U.S.-Asia gap in STEM interest was explained by perceptions that STEM provided communal and agentic opportunities. We employed path analyses using maximum likelihood estimation exploring indirect effects using bootstrapped standard errors (Hayes, 2013). As shown in Figure 2, all studies demonstrated that students from Asian countries were more likely to perceive STEM as providing communal opportunities, and perceived communal opportunities predicted STEM interest (Study 1: IE 0.219, 95\% CI [0.497, 0.978]; Study 2: IE 0.079, 95\% CI [0.074, 0.308]; Study
3: IE $0.143,95 \%$ CI [0.157, 0.438]; Study 4: IE 0.043, 95\% CI $[0.036,0.207])$. Although perceived agentic opportunities also predicted interest, this perception cannot explain the U.S.-Asia gap because no country differences in perceived agentic opportunities emerged.

\section{What Were the Sources of Perceptions About Communal and Agentic Opportunities in STEM?}

Because Asian students perceived that STEM careers provided more communal opportunities, and communal and agentic opportunities predicted STEM interest, we examined three possible sources for differential perceptions of STEM: (1) a general tendency by Asian, as compared with U.S., students to perceive that many careers provided communal opportunities; (2) Asian, as compared with U.S., students completed more STEM classes, fostering perceptions that STEM provided more agentic and communal opportunities; and (3) Asian, as compared with U.S., students reported more communal engagement in STEM educational experiences, fostering perceptions that STEM provided more communal opportunities.

Cultural differences in the general tendency to perceive communal opportunities in careers. In Studies 1 and 3, we examined whether Asian, as compared with U.S., students perceived that many different occupations, not just STEM, provided more communal opportunities by conducting 2 (country) $\times 2$ (perceived opportunities: communal, agentic) $\times 3$ (career type: STEM, male-stereotypic, female-stereotypic) mixed ANOVAs with country as a between-subjects variable. As predicted, significant Country $\times$ Perceived Opportunities $\times$ Career Type interactions emerged, Study 1: $F(2,684)=105.26, p<.001$; Study 3: $F(2,510)=49.50, p<.001$.

We decomposed the three-way interactions by examining the Country $\times$ Career Type interactions within communal and agentic opportunities. The significant Country $\times$ Career Type interactions emerged for perceived communal opportunities, Study 1: $F(2,684)=77.44, p<.001$; Study $3: F(2,510)=$ 


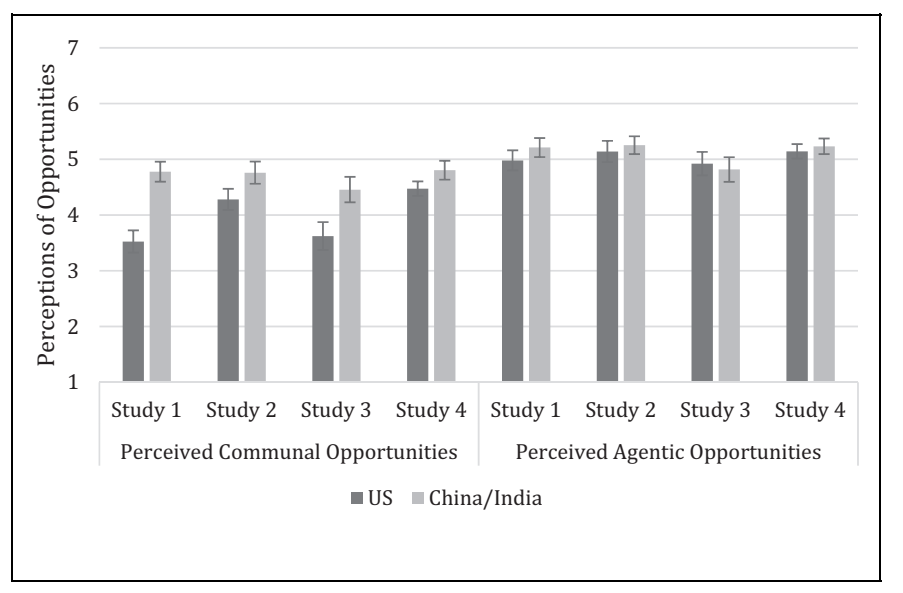

Figure I. Country differences in perceptions of communal and agentic opportunities in science, technology, engineering, and mathematics-Studies 1-4. Perceived opportunities were rated on 7-point scales. The error bars represent $95 \%$ confidence intervals.

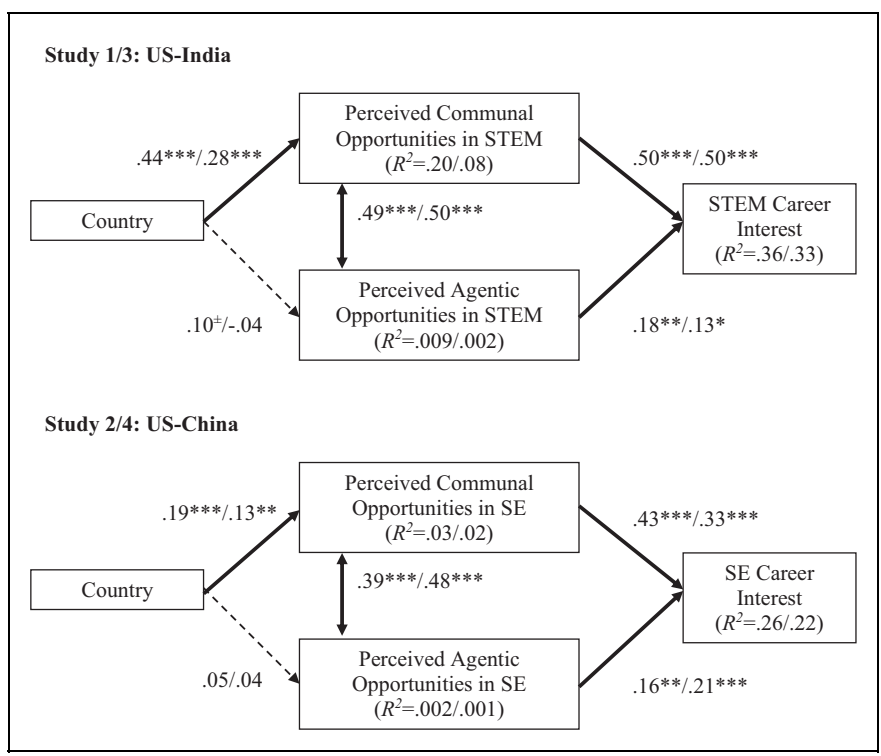

Figure 2. Perceptions of communal opportunities in science, technology, engineering, and mathematics (STEM) uniquely predicted the cultural difference in STEM career interest. Country was dummy coded as Asian Country Status = I; U.S. Country Status = 0. Path coefficients represent significant standardized estimates. Dashed lines represent nonsignificant model paths. $*_{p}<.05$. $*_{p}<.01$. $*_{* *}<.001$. $\pm p<.10$.

53.26, $p<.001$. Contrary to the explanation positing that Asian students perceive all careers as providing more communal opportunities, the U.S. and Asian students only differed consistently on perceptions of STEM. Asian students were more likely than U.S. students to perceive STEM careers as providing communal opportunities (Study 1: $p<.001, d=0.99, \mathrm{CI}$ $[0.857,1.125]$; Study 3: $p<.001, d=0.60$, CI [0.424, 0.766]). However, U.S. students were more likely than Asian students to perceive female-stereotypic (Study 1: $p=.009$, $d=-0.29$, CI $[-0.401,-0.171]$; Study 3: $p<.001$, $d=-0.48$, CI $[-0.622,-0.342])$ and, in one case,

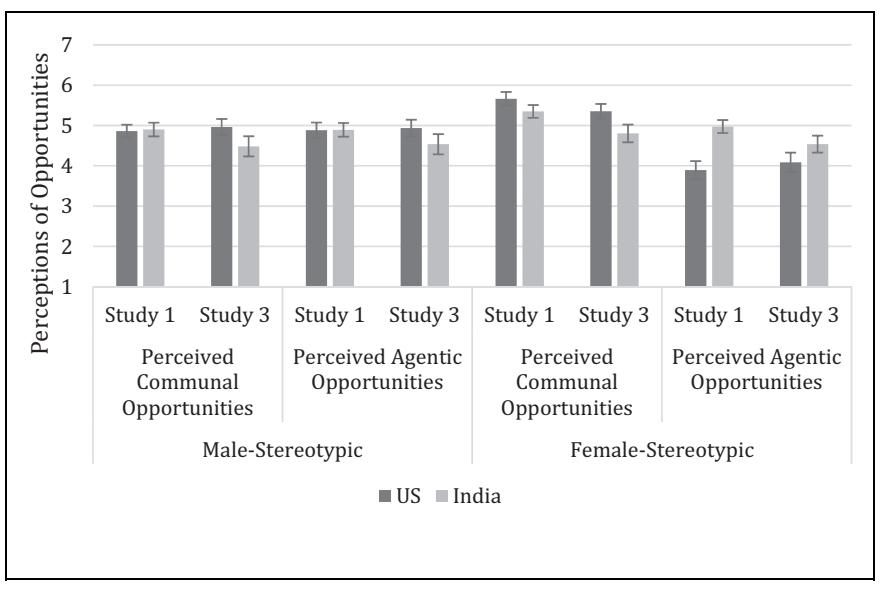

Figure 3. Country differences in perceptions of communal and agentic opportunities in male-stereotypic and female-stereotypic careers-Studies I and 3. Perceived opportunities were rated on 7 -point scales. The error bars represent $95 \%$ confidence intervals.

male-stereotypic (Study 3: $p=.003, d=-0.37$, CI $[-0.531$, $-0.217]$ ) careers as providing communal opportunities.

The significant Country $\times$ Career Type interaction for perceived agentic opportunities showed a different pattern (Study $1: F[2,684]=45.32, p<.001$; Study $3: F[2,510]=18.51$, $p<.001)$. Asian more than U.S. students perceived that female-stereotypic careers provided agentic opportunities (Study 1: $p<.001, d=0.85$, CI [0.721, 0.988]; Study 3: $p=.006, d=0.35$, CI $[0.187,0.505])$. Other effects did not replicate across studies: In Study 3, U.S. more than Asian students perceived that male-stereotypic careers provided agentic opportunities ( $p=.015, d=-0.31$, CI $[-0.466,-0.149])$; in Study 1, Asian more than U.S. students perceived that STEM careers provided agentic opportunities $(p=.073, d=0.20$, CI [0.071, 0.320]; see Figure 3). Because these effects were not predicted, we hesitate to interpret them strongly.

Cultural differences in the quantity of STEM classes. In Studies 3 and 4, we examined participants' self-reported history of number of STEM classes as one source of differential perceived goal opportunities in STEM. We first established whether country differences emerged: As predicted, a one-way ANOVA revealed that Asian students reported taking more classes in STEM, Study 3: $F(1,246)=34.72$, $p<.001, d=0.76$, CI $[-0.950,2.460]$; Study 4: $F(1$, $551)=32.26, p<.001, d=0.50$, CI $[-0.147,1.146]$, than U.S. students.

Cultural differences in communal engagement in STEM. We also examined country differences in communal engagement in math and science classes. As predicted, a one-way ANOVA revealed that Asian students reported having more communal experiences in STEM, Study 3: $F(1,251)=63.30, p<.001$, $d=1.01$, CI $[0.817,1.199]$; Study $4: F(1,553)=138.31$, $p<.001, d=1.03$, CI $[0.921,1.144]$, than U.S. students. 


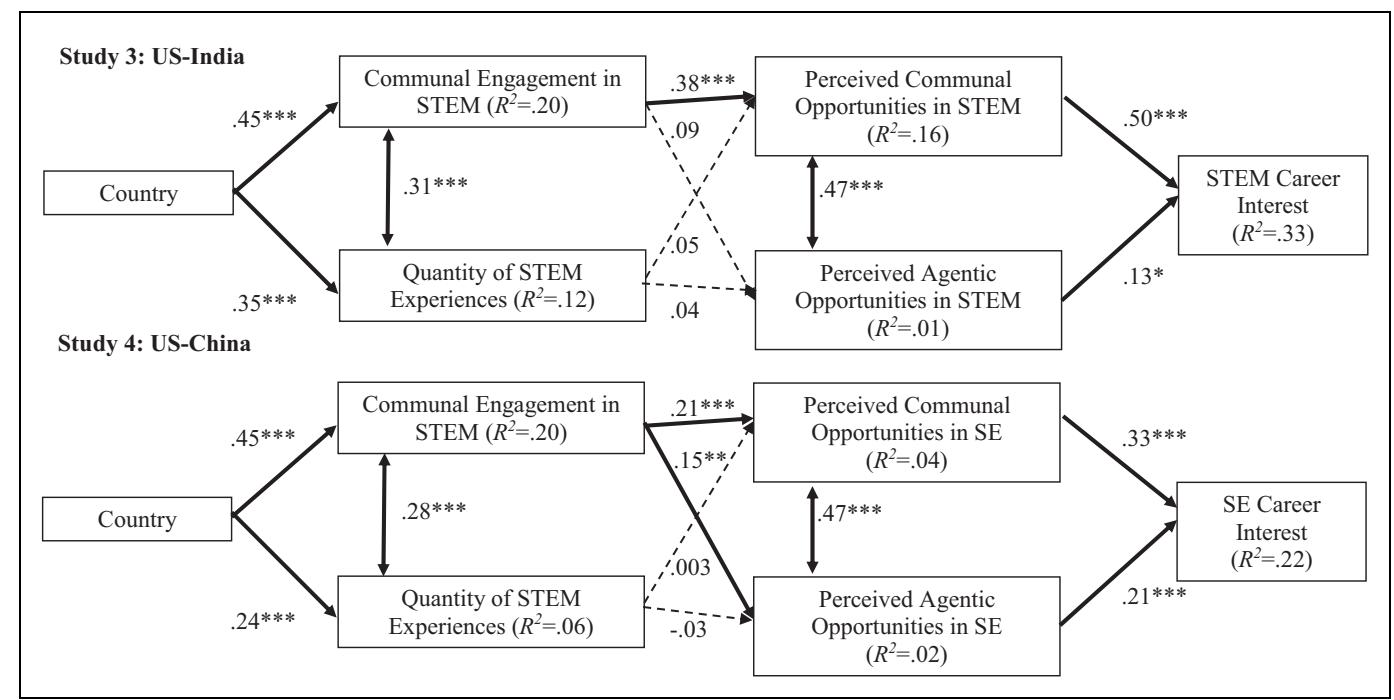

Figure 4. Communal goal experiences and perceptions of communal opportunities in science, technology, engineering, and mathematics (STEM) predict the cultural difference in STEM career interest. Country was dummy coded as Asian Country Status = I; U.S. Country Status =0. Path coefficients represent significant standardized estimates. Dashed lines represent nonsignificant model paths. ${ }^{*} p<.05$. ${ }^{*} p<.0 \mathrm{I}$. $* * * p<.001$.

Did cultural differences in number of STEM classes and/or communal engagement in STEM underlay the U.S.-Asia gap in STEM interest? We next examined whether country differences in communal engagement and/or an increased number of classes taken in STEM accounted for increased perceptions that STEM provided communal or agentic opportunities, which subsequently predicted increased interest in STEM. We submitted variables to path analyses with maximum likelihood estimation and examined indirect effects using bootstrapped standard errors (Hayes, 2013). Asian student status predicted engaging in more communal experiences in STEM as well as taking more STEM classes. However, communal experience in STEM uniquely predicted perceived communal opportunities in STEM (and in Study 4, perceived agentic opportunities as well). Lastly, perceptions that STEM provided communal and agentic opportunities predicted increased interest in STEM careers (indirect effect through communal experiences in STEM and perceived communal opportunities: Study 3: IE $0.086,95 \%$ CI [0.150, 0.471]; Study 4: IE $0.030,95 \%$ CI [0.030, 0.132]; unexpected indirect effect through communal experiences in STEM and perceived agentic opportunities: Study 4: IE 0.015, 95\% CI [0.011, 0.075]; see Figure 4).

\section{Discussion}

Across four studies, Asian students, relative to U.S. students, reported more interest in STEM, perceived that STEM provided more communal opportunities, reported taking more STEM classes, and reported more communal experiences in STEM. Furthermore, the U.S.-Asia gap in interest was explained not by a general tendency to perceive all careers as providing communal or agentic opportunities or by taking more STEM-related classes, but by greater communal experiences within STEM fields. These communal experiences in STEM were associated with increased perceptions that STEM provided communal opportunities and ultimately accounted for more interest in engaging in STEM.

Although communal experiences were an important contributor to the U.S.-Asia gap in STEM perceptions and interest, inferences are limited by the correlational nature of these studies. Study 5 addresses this limitation by examining whether experimentally manipulating perceived communal opportunities within the domain of science closed the U.S.-Asia gap. Studies 1-4 demonstrated that Asian students, as compared with U.S. students, perceived science as providing higher levels of communal opportunities. Thus, in Study 5, we did not anticipate that additional exposure to the perceived communal opportunities in science would further increase Asian participants' perceptions because this information is consistent with preexisting beliefs. However, we predicted that exposure to communal opportunities in science would have a unique impact on U.S. participants because this information is counterstereotypic. We anticipated that exposure to communal information in science would increase U.S. participants' perceptions of the communal opportunities provided by science and their positivity toward a scientist career.

\section{Study 5}

\section{Method}

\section{Sample Size}

We collected at least 73 participants per country (the minimum needed for .85 power and a moderate effect size). We oversampled to allow for weaker effect sizes and supplementary analyses (see Supplementary Material). 


\section{Participants}

One hundred fifty-two Indian participants (59 women, 93 men; ages $21-78[m d n=30])$ and 175 U.S. participants (103 women, $71 \mathrm{men}, 1$ unspecified; ages $18-73[m d n=32])$ were recruited for payment through MTurk.

\section{Procedure}

In an online survey in English, participants read about the typical day of a scientist (communal or noncommunal), rated their perceptions of the communal and agentic opportunities provided by a scientist career, and rated their positivity toward science, resulting in a 2 (country) $\times 2$ (framing) between-subjects design.

\section{Independent Variables}

Participants were randomly assigned to read about the typical day of a scientist who completed eight tasks, six of which were framed as being either communal (i.e., working with others) or noncommunal (i.e., independent work; Diekman et al., 2011; see Figure 5).

\section{Dependent Variables}

Perceived communal and agentic opportunities. Similar to Studies $1-4$, participants rated how much a scientist career provided communal ("such as intimacy, affiliation, and altruism") and agentic ("such as power, achievement, and seeking new experiences or excitement") opportunities on scales ranging from 1 (not at all) to 7 (extremely; modified from Diekman et al., 2010).

Science positivity. Participants rated their general impression and their projected enjoyment of a scientist career on scales ranging from 1 (not at all) to 7 (extremely). These items were averaged $(\alpha=.70$; modified from Diekman et al., 2011).

\section{Results}

First, we confirmed that the communal framing manipulation caused U.S. but not Indian participants to perceive that a scientist career provided communal opportunities. Next, we examined whether exposure to the communal framing manipulation led U.S. participants to express more positivity toward a scientist career than Indian participants. Finally, we conducted separate path analyses for U.S. and Indian participants, examining whether exposure to the framing manipulation increased perceptions of communal and agentic opportunities in science and subsequently increased positivity toward a scientist career. For clarity, we report results relevant to our key hypotheses. Complete ANOVA results are available in Tables 4 and 5.

\section{Perceived Opportunities to Fulfill Communion and Agency}

We conducted a 2 (country) $\times 2$ (framing: communal, agentic) $\times 2$ (perceived opportunities: communal, agentic) mixed ANOVA with perceived opportunities as a within-subjects variable. The predicted Country $\times$ Perceived Opportunities $\times$ Framing interaction emerged, $F(1,323)=15.87, p<.001$. For perceptions that science provided communal opportunities, a significant Country $\times$ Framing interaction emerged, $F(1,323)=22.61, p<.001$. As expected, among U.S. participants, reading about the communal versus noncommunal day of a scientist increased the perception that science careers provided communal opportunities $(p<.001, d=$ 1.05 , CI $[0.859,1.246])$. Among Indian participants, reading about the communal versus noncommunal day of a scientist did not affect the perception that science careers provided communal opportunities $(p=.770, d=0.05$, CI $[-0.137$, 0.233]; see Figure 6).

Science positivity. To examine whether exposure to the communal framing manipulation led U.S. participants to express more positivity toward a scientist career than Indian participants, we conducted a 2 (country) $\times 2$ (framing) between-subjects ANOVA. Importantly, participants who read about the communal versus noncommunal day of a scientist expressed greater positivity toward a scientist career, $F(1,323)=4.93, p=$ $.027, d=0.26$, CI $[0.125,0.403]$. Although the Country $\times$ Framing interaction did not emerge, $F(1,323)=0.79, p=$ .375 , simple effects were consistent with hypotheses. Among U.S. participants, reading about the communal $(M=5.19$, $S D=1.32)$ versus noncommunal $(M=4.76, S D=1.43)$ day of a scientist increased positivity toward a scientist career $(p=.039, d=0.32$, CI $[0.115,0.520])$. Among Indian participants, reading about the communal $(M=5.63, S D=1.03)$ versus noncommunal $(M=5.44, S D=1.18)$ day of a scientist did not affect positivity toward a scientist career $(p=.302$, $d=0.17$, CI $[-0.005,0.344])$.

Did perceptions about communal and agentic opportunities underlay the effect of communal framing on positivity toward a scientist career? To examine whether perceptions that science provided communal opportunities underlay the communal framing effect on positivity toward a scientist career, we conducted separate path analyses with maximum likelihood estimation within the U.S. and Indian groups. We used bootstrapped standard errors to examine indirect effects (Hayes, 2013). Consistent with our hypotheses, for U.S. participants, reading about the communal versus the noncommunal day of a scientist increased perceptions that science provided communal opportunities; these perceived communal opportunities predicted positivity toward a scientist career (indirect effect through communal opportunity perceptions: IE $0.105,95 \%$ CI [0.097, 0.531]). Unexpectedly, the communal framing also fostered agentic perceptions, which, in turn, predicted positivity toward a scientist career (indirect effect through agentic opportunity perceptions: IE $0.049,95 \%$ CI $[0.005,0.298])$. No significant indirect effects occurred for Indian participants (indirect through communal opportunity perceptions: IE $0.010,95 \%$ CI $[-0.107,0.204]$; indirect effect through agentic opportunity perceptions: IE $0.029,95 \%$ CI $[-0.056,0.190]$; see Figure 7 ). 
Communal framing

8:I5 am

I come in and check my e-mail then plan the day. I usually have to communicate closely with the Operations Group (they run the high-throughput screens) to check on the status of ongoing experiments so we can go from primary to secondary characterizations.

9:15 am

I go to the lab after about an hour to check on samples left overnight (for example, to see if a drug crystallized), characterize samples from the previous afternoon to integrate the data collected the previous day, and characterize new samples that have come in that day. I meet some of my lab group in the lab and consult with them about the procedures.

12:00 pm

I join co-workers from other labs at lunch. The company runs presentations during lunch, where we learn what else is going on both within the company and with the Big Pharma companies who supply us with compounds. Speakers might be a group member from a different group giving an update, a patent lawyer briefing us on legal issues in patent protection, and a member of the Products Group describing ongoing product development work. Lunch is a good chance to catch up on the progress that other labs are making, and to share our ideas and feedback.

$\mathrm{I}: 00 \mathrm{pm}$

Mentor new members of my statistics group in doing data analysis (e.g., powder X-ray diffraction, differential scanning calorimetry, thermal gravimetric analysis).

3:00 pm

Collaborate with my group (which has 6 members) to prepare for a meeting with our supervisor. Go to meeting to update our supervisor on the status of our projects, which are typically larger projects that have several team members. Our supervisor will ask questions and give advice on running further experiments or collecting additional data points. Our supervisor also gives us a heads-up on what compounds are coming in during the next few weeks. This gives us an idea of the workload of the group.

4:00 pm

Update lab notebook with either data collected that day or experiments started. Get started on experiments that can be set up and run overnight.

5:00 pm

Prepare for the monthly presentation my lab group gives at local schools to inform interested students about our research. Typically, I make a PowerPoint presentation using tables and charts of data, a summary, and discussion points.

\section{5:30 pm}

Commute home.

I like that so much of my work involves working closely with other people and helping them solve problems. The interactions we have are really fun, and I get the sense that I am contributing a great deal to their projects. I like having a variety of tasks, gathering data through multiple methods, and trying to interpret data from both high-throughput experiments and bench-top experiments. I like the sense of contributing to understanding drug candidates that are likely to get into clinical trials. I love that my job can have a positive impact on so many people.

Noncommunal framing

8:15 am

I come in and check my e-mail then plan the day. I usually have to check a database maintained by the Operations Group (they run the highthroughput screens) to learn the status of ongoing experiments so I can go from primary to secondary characterizations.

9:15 am

I go to the lab after about an hour to check on samples left overnight (for example, to see if a drug crystallized), characterize samples from the previous afternoon to integrate the data collected the previous day, and characterize new samples that have come in that day. I look up relevant past research to consult about the procedures.

12:00 pm

The company runs presentations during lunch, where we learn what else is going on both within the company and with the Big Pharma companies who supply us with compounds. I watch video feed of these presentations at my desk while I eat. Speakers might be a researcher from a different lab giving an update, a patent lawyer briefing us on legal issues in patent protection, and a member of the Products Group describing ongoing product development work.

I:00 pm

Do data analysis (e.g., powder X-ray diffraction, differential scanning calorimetry, thermal gravimetric analysis) and troubleshoot any problems that come up by myself.

3:00 pm

Go to meeting to update my supervisor on the status of my projects, which are typically independent. My supervisor will tell me what further experiments to run or additional data points to collect. My supervisor also gives me a heads-up on what compounds are coming in during the next few weeks. This gives me an idea of what my own workload will be like.

Figure 5. Framing of a typical day of a scientist-Study 5. 
4:00 pm

Update lab notebook with either data collected that day or experiments started. Get started on experiments that can be set up and run overnight.

5:00 pm

Prepare for weekly meetings with the entire Solid State Chemistry Group (I5 members). Typically, I make a PowerPoint presentation using tables and charts of data, a summary, and discussion points.

5:30 pm

Commute home.

I like that so much of my work involves working by myself and solving problems. The solitary nature of my work is really fun, and I get the sense that I am achieving a great deal through my projects. I like having a variety of tasks, gathering data through multiple methods, and trying to interpret data from both high-throughput experiments and bench-top experiments. I like the sense of achievement I have in my current position. I love that my job provides me with intellectual challenge and financial security.

Figure 5. (continued).

Table 4. Perceptions of Communal and Agentic Opportunities in Science-Study 5. ${ }^{\mathrm{a}}$

2 (country: United States, China/India) $\times 2$ (perceived opportunities: communal, agentic) $\times 2$ (framing: communal, agentic) ANOVA

\begin{tabular}{lrrr}
\hline & \multicolumn{3}{c}{ Study 5 (df: I, 323) } \\
\cline { 2 - 5 } & \multicolumn{1}{c}{$f$} & $p$ & $d$ \\
\hline Country & 23.39 & $<.001$ & .53 \\
Perceived opportunities & 2.03 & .155 & -.18 \\
Framing & 18.24 & $<.001$ & .50 \\
Country $\times$ Perceived Opportunities & 0.05 & .820 & - \\
Country $\times$ Framing & 10.54 & .001 & - \\
Perceived Opportunities $\times$ Framing & 8.96 & .003 & - \\
Country $\times$ Perceived Opportunities $\times$ Framing & 15.87 & $<.001$ & - \\
$\quad$ United States & & & \\
$\quad$ Perceived communal opportunities & & & \\
$\quad$ Framing & 47.85 & $<.001$ & 1.05 \\
$\quad$ Perceived agentic opportunities & & & \\
$\quad$ Framing & 4.43 & .037 & .31 \\
China/lndia & & & \\
$\quad$ Perceived communal opportunities & & & \\
$\quad$ Framing & 0.09 & .770 & .05 \\
$\quad$ Perceived agentic opportunities & & & \\
$\quad$ Framing & 1.16 & .283 & .18 \\
\hline
\end{tabular}

Note. ANOVA = analysis of variance.

aPositive effect sizes (Cohen's $d$ ) indicate higher ratings for Asian participants, perceived communal opportunities, and communal framing.

\section{Discussion}

Consistent with our hypotheses, reading about the communally oriented work of a scientist affected U.S. but not Indian participants' beliefs and attitudes about science. Exposure to information that science can include communal opportunities offers new information for U.S. participants, whereas this information is consistent with the existing perceptions of STEM held by Indian participants (see Studies $1-4$ ). It is possible that a different type of manipulation might highlight communal opportunities that are perceived as missing in STEM even among Asian participants and that this information would increase perceived communal opportunities for
Table 5. Positivity Toward Science-Study 5.

2 (country: United States, China/India) $\times 2$ (framing: communal, agentic) ANOVA

\begin{tabular}{lccc}
\hline & \multicolumn{3}{c}{ Study 5 (df: I, 323) } \\
\cline { 2 - 4 } & $F$ & $p$ & $d$ \\
\hline Country & 15.88 & $<.001$ & .45 \\
Framing & 4.93 & .027 & .26 \\
Country $\times$ Framing & 0.79 & .375 & - \\
$\quad$ United States & & & \\
$\quad$ Framing & 4.35 & .039 & .31 \\
China/India & & .302 & .17 \\
$\quad$ Framing & 1.07 & & \\
\hline
\end{tabular}

Note. ANOVA = analysis of variance.

a'Positive effect sizes (Cohen's $d$ ) indicate higher ratings for Asian participants and communal framing.

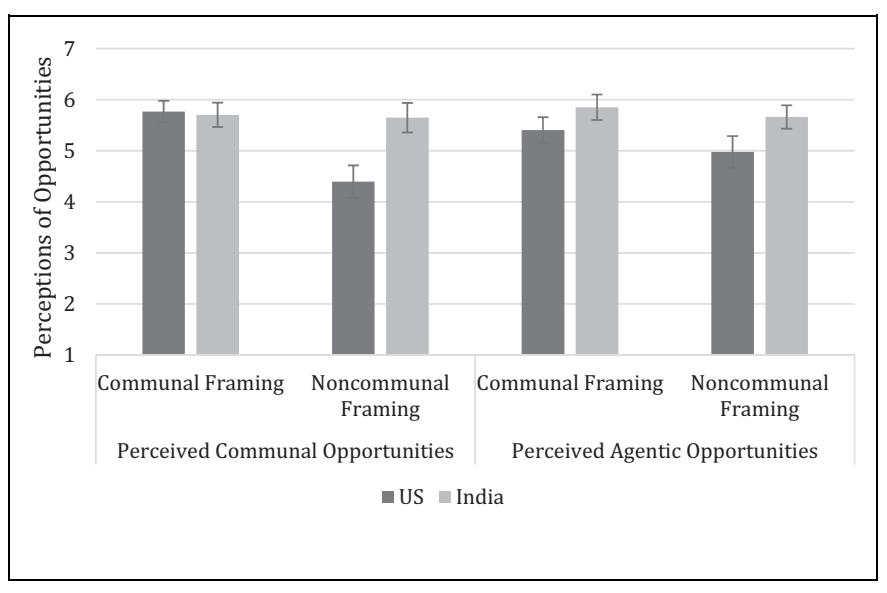

Figure 6. Experimental effects of framing on perceived communal and agentic opportunities in science careers-Study 5. Perceived opportunities were rated on 7 -point scales. The error bars represent $95 \%$ confidence intervals.

Asian participants as well. The current data provide evidence the causal role of aligning perceived role opportunities with valued goals. If the aim is to foster perceptions that might 


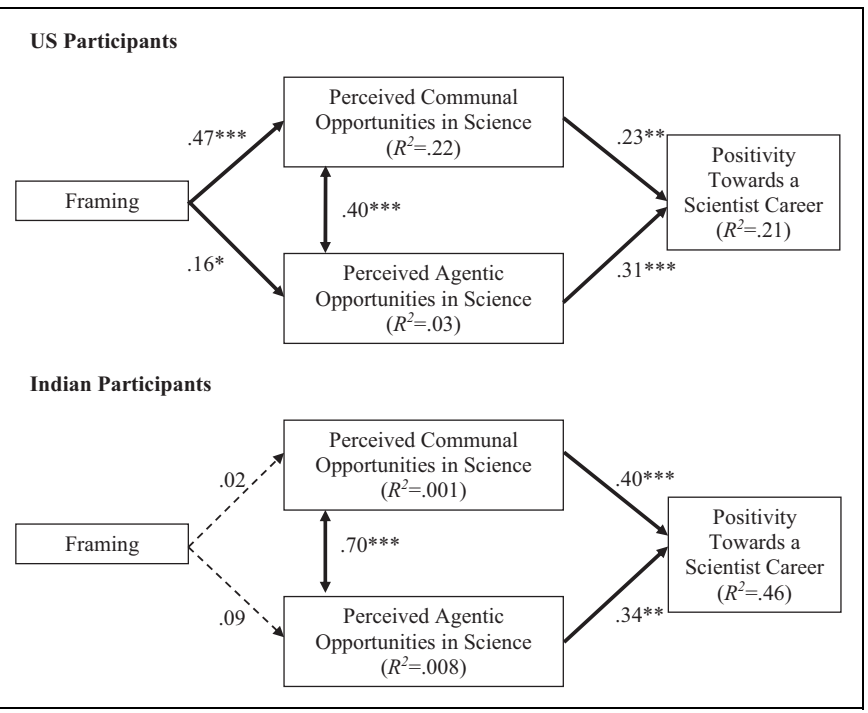

Figure 7. Do perceptions of communal and agentic opportunities in science differentially underlie the framing effect on positivity toward science careers for US and Indian participants?-Study 5. Framing was dummy coded as communal $=\mathrm{I}$; agentic $=0$. Path coefficients represent significant standardized estimates. Dashed lines represent nonsignificant model paths. ${ }^{* *} p<.001 ; *^{*} p<.01 ; * p<.05$.

draw U.S. students into the STEM pathway, including communal information may be one way to provide more equal footing with Asian students.

\section{General Discussion}

Engaging U.S. students' communal goals within the context of STEM offers a new vantage point to promote engagement within the STEM pipeline. Across multiple samples and methodologies, U.S. participants perceived fewer opportunities than did Asian participants to fulfill communal goals in STEM, and this differential perception contributed to U.S.-Asian gaps in positivity toward engaging in STEM. Individuals who perceived STEM as offering more opportunities to work with or help others reported greater positivity toward STEM. Furthermore, highlighting communal opportunities within a scientist's workday increased U.S., and not Asian, participants' perceptions that science provided more communal and agentic opportunities, and these opportunities were linked with increased positivity toward a scientist career.

Understanding communal processes in the context of STEM pathways can increase and maintain participation in the STEM pipeline. Highlighting the perceived communal opportunities within STEM increases the attractiveness of STEM to U.S. participants generally (Brown, Smith, et al., 2015) as well as to the members of underrepresented groups (girls: Weisgram \& Bigler, 2006; women: Diekman et al., 2011; and underrepresented minority students: Thoman et al., 2015). Furthermore, biomedical research assistants who perceived that science provided communal opportunities reported increased science motivation and positivity over time (Brown, Smith, et al., 2015). Thus, emphasizing communal opportunities can open the doors to STEM more broadly, without alienating or discouraging others. This broader appeal of STEM is important, given the need to increase the overall quality and quantity of STEM talent.

A limitation of the current research is that the challenges of cross-cultural research did not allow us to explore alternative or more elaborated models. For example, communal experiences in classes might heighten the success of students, which, in turn, might predict interest in SE careers. Indeed, other U.S. data (Fuesting, Diekman, \& Hudiburgh, 2017) indicate that exposure to communal applications of material in STEM classes predicted positivity toward and greater selfreported success in STEM classes. However, beyond these effects, exposure to communal applications independently predicted beliefs that STEM careers would fulfill communal goals, and beliefs about communal opportunities in STEM more robustly predicted interest in pursuing those careers than did academic positivity and success. We hope that the current research opens the door to explore how communal opportunities in STEM can particularly engage students to succeed and persist, and that empirical answers to how communal opportunities and success foster STEM interest and persistence will accumulate. We certainly do not contend that highlighting communal opportunities in SE is the only route to closing U.S.-Asia STEM gaps, but we do contend that understanding and addressing these communal perceptions is a useful and underutilized tool in explaining and possibly alleviating the U.S.-Asia gap in STEM interest.

Integrating communal opportunities in STEM (i.e., providing opportunities to work with others; designing activities that help the community) provides a concrete pathway to increasing students' motivation to engage in higher quantities of intensive science and mathematics. More exposure or higher standards for STEM education only reap benefits when students are motivated to immerse themselves in the rigorous work of science and mathematics. For a problem as challenging, complex, and important as increasing the quantity and quality of the STEM workforce, multiple evidence-based solutions are needed, and highlighting communal opportunities within STEM offers one potential way to both broaden and deepen STEM participation in the United States.

\section{Authors' Note}

Elizabeth R. Brown's first authorship was determined by a coin toss.

\section{Acknowledgments}

We thank Mingzheng $\mathrm{Wu}$ for recruiting the Chinese samples and Aimee Belanger, Tessa Benson-Greenwald, Emily Clark, Melissa Fuesting, Kurt Hugenberg, Vrinda Kalia, Vaishali Raval, and the UNF Psychology Writing Group for their comments on drafts of the article.

\section{Declaration of Conflicting Interests}

The author(s) declared no potential conflicts of interest with respect to the research, authorship, and/or publication of this article. 


\section{Funding}

The author(s) disclosed receipt of the following financial support for the research and/or authorship of this article: National Science Foundation (NSF)/Research on Gender in Science and Engineering (GSE) 1232364 and NSF/GSE 0827606 to Amanda B. Diekman.

\section{Supplemental Material}

The supplemental material is available in the online version of the article.

\section{Notes}

1. In Studies 2 and 4, we collected data from additional samples of U.S. participants for payment using MTurk. Analyses using the U.S. MTurk participants (in lieu of the U.S. college students) as compared with the Chinese college students yielded similar results and are explained in the Supplementary Material.

2. Due to a programming error, registered nurse was rated only in Study 1.

\section{References}

Bakan, D. (1966). The duality of human existence: An essay on psychology and religion. Chicago, IL: Rand McNally.

Baumeister, R. F., \& Leary, M. R. (1995). The need to belong: Desire for interpersonal attachments as a fundamental human motivation. Psychological Bulletin, 117, 497-529. doi:10.1037/0033-2909. 117.3.497

Brinkman, B., \& Diekman, A. (2016). Applying the communal goal congruity perspective to enhance diversity and inclusion in undergraduate computing degrees. In Proceedings of the 47th ACM Technical Symposium on Computing Science Education (pp. 102-107). New York, NY: ACM. doi:10.1145/2839509. 2844562

Brown, E. R., Smith, J. L., Thoman, D. B., Allen, J. M., \& Muragishi, G. (2015). From bench to bedside: A communal utility value intervention to enhance students' biomedical science motivation. Journal of Educational Psychology, 107, 1116-1135. doi:10.1037/ edu0000033

Brown, E. R., Thoman, D. B., Smith, J. L., \& Diekman, A. B. (2015). Closing the communal goal gap: Degree of goal congruity predicts science career motivation. Journal of Applied Social Psychology, 45, 662-673. doi:10.1111/jasp. 12327

Clark, E. K., Fuesting, M. A., \& Diekman, A. B. (2016). Enhancing interest in science: Exemplars as cues to communal affordances of science. Journal of Applied Social Psychology, 46, 641-654. doi:10.1111/jasp.12392

Colvin, W., Lyden, S., \& León de la Barra, B. (2013). Attracting girls to civil engineering through hands-on activities that reveal the communal goals and values of the profession. Leadership and Management in Engineering, 13, 35-41. doi:10.1061/ (ASCE)LM.1943-5630.0000208

Diekman, A. B., Brown, E. R., Johnston, A. M., \& Clark, E. K. (2010). Seeking congruity between goals and roles: A new look at why women opt out of science, technology, engineering, and mathematics careers. Psychological Science, 21, 1051-1057. doi:10.1177/0956797610377342
Diekman, A. B., Clark, E. K., Johnston, A. M., Brown, E. R., \& Steinberg, M. (2011). Malleability in communal goals and beliefs influences attraction to STEM careers. Journal of Personality and Social Psychology, 101, 902-918. doi:10.1037/a0025199

Diekman, A. B., Steinberg, M., Brown, E. R., Belanger, A. L., \& Clark, E. K. (2016). A goal congruity model of role entry, engagement, and exit: Understanding communal goal processes in STEM gender gaps. Personality and Social Psychology Review. doi:10. 1177/1088868316642141

Eccles, J. S., \& Wigfield, A. (1995). In the mind of the actor: The structure of adolescents' achievement task values and expectancy-related beliefs. Personality and Social Psychology Bulletin, 21, 215-225. doi:10.1177/0146167295213003

Fiske, S. T. (2004). Social beings: A core motives approach to social psychology. Hoboken, NJ: Wiley.

Fuesting, M. A., Diekman, A. B., \& Hudiburgh, L. (2017). From classroom to career: The unique role of communal processes in predicting interest in STEM careers. Manuscript in preparation.

Harackiewicz, J. M., Smith, J. L., \& Priniski, S. J. (2016). Interest matters: The importance of promoting interest in education. Policy Insights from the Behavioral and Brain Sciences. doi:10.1177/ 2372732216655542

Hayes, A. F. (2013). Introduction to mediation, moderation, and conditional process analysis: A regression-based approach. New York, NY: Guilford Press.

Hines, P. J., Mervis, J., Mccartney, M., \& Wible, B. (2013). Plenty of challenges for all. Science, 340, 290-291. doi:10.1126/science. 340.6130.290

Markus, H. R., \& Kitayama, S. (1991). Culture and the self: Implications for cognition, emotion, and motivation. Psychological Review, 98, 224-253. doi:10.1037/0033-295X.98.2.224

National Science Board. (2012). Science and Engineering Indicators 2012. Arlington, VA: National Science Foundation (NSB 12-01).

National Science Board. (2014). Science and Engineering Indicators 2014. Arlington, VA: National Science Foundation (NSB 14-01).

National Science Board. (2016). Science and Engineering Indicators 2016. Arlington, VA: National Science Foundation (NSB 2016-01).

Pohlmann, K. (2001). Agency- and communion-orientation in life goals: Impacts on goal pursuit strategies and psychological wellbeing. In P. Schmuck \& K. M. Sheldon (Eds.), Life goals and well-being: Towards a positive psychology of human striving (pp. 68-84). Seattle, WA: Hogrefe \& Huber.

President's Council of Advisors on Science and Technology. (2010). Prepare and inspire: K-12 Science, technology, engineering, and math (STEM) education for America's future. Washington, DC. Retrieved from http:/www.whitehouse.gov/sites/default/files/ microsites/ostp/pcast-stemed-report.pdf

Ryan, R. M., \& Deci, E. L. (2000). Intrinsic and extrinsic motivation: Classic definitions and new directions. Contemporary Educational Psychology, 25, 54-67. doi:10.1006/eeps.1999.1020

Smith, J. L., Brown, E. R., Thoman, D. B., \& Deemer, E. D. (2015). Losing its expected communal value: How stereotype threat undermines women's identity as research scientists. Social Psychology Education, 18, 443-466. doi:10.1007/s11218-015-9296-8

Steinberg, M. (2011). Working together to increase STEM interest: Communal experience and its effect on the malleability of STEM 
beliefs and attitudes. Miami University. Retrieved from https://etd. ohiolink.edu/ap/10?0::NO:10:P10_ACCESSION_NUM: miami1321555792

Talhelm, T., Zhang, X., Oishi, S., Shimin, C., Duan, D., Lan, X., \& Kitayama, S. (2014). Large-scale psychological differences within China explained by rice versus wheat agriculture. Science, 344, 603-608. doi:10.1126/science. 1246850

Thoman, D. B., Brown, E. R., Mason, A. Z., Harmsen, A. G., \& Smith, J. L. (2015). The role of altruistic values in motivating underrepresented minority students for biomedicine. BioScience, 65, 183-188. doi:10.1093/biosci/biu199

U.S. Congress Joint Economic Committee. (2012). STEM education: Preparing for the jobs of the future. Washington, DC: Author.

Uskul, A. K., Kitayama, S., \& Nisbett, R. E. (2008). Ecocultural basis of cognition: Farmers and fishermen are more holistic than herders. Proceedings of the National Academy of Sciences, 105, 8552-8556. doi:10.1073/pnas.0803874105

Valla, J. M., \& Ceci, S. J. (2014). Breadth-based models of women's underrepresentation in STEM fields. Perspectives on Psychological Science, 9, 219-224. doi:10.1177/1745691614522067
Varnum, M. E. W., Grossmann, I., Kitayama, S., \& Nisbett, R. E. (2010). The origin of cultural differences in cognition the social orientation hypothesis. Current Directions in Psychological Science, 19, 9-13. doi:10.1177/0963721409359301

Weisgram, E. S., \& Bigler, R. S. (2006). The role of attitudes and intervention in high school girls' interest in computer science. Journal of Women and Minorities in Science and Engineering, 12, 325-336. doi:10.1615/JWomenMinorScienEng. v12.i4.40

\section{Author Biographies}

Elizabeth R. Brown is an assistant professor of psychology.

Mia Steinberg is a postdoctoral fellow.

Yun Lu is a doctoral student in counseling psychology.

Amanda B. Diekman is a professor of psychology.

Handling Editor: Nickola Overall 